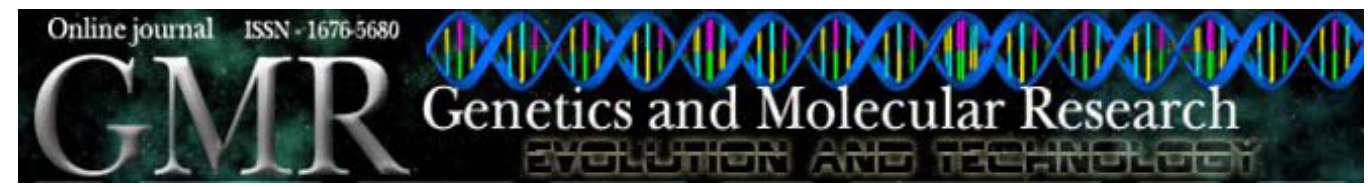

Case Report

\title{
Cytogenetic findings in an epithelioid sarcoma with angiomatoid features. A case report
}

\author{
M.S. Brassesco ${ }^{1}$, E.T. Valera ${ }^{1}$, A.M. Castro-Gamero ${ }^{2}$, D.A. Moreno ${ }^{2}$, \\ T.P. Silveira ${ }^{3}$, B.M. Mori ${ }^{1}$, E.E. Engel ${ }^{4}$, C.A. Scrideli $^{1}$ and L.G. Tone ${ }^{1}$ \\ ${ }^{1}$ Departamento de Puericultura e Pediatria, Faculdade de Medicina de \\ Ribeirão Preto, Universidade de São Paulo, Ribeirão Preto, SP, Brasil \\ ${ }^{2}$ Departamento de Genética, Faculdade de Medicina de Ribeirão Preto, \\ Universidade de São Paulo, Ribeirão Preto, SP, Brasil \\ ${ }^{3}$ Departamento de Patologia, Faculdade de Medicina de Ribeirão Preto, \\ Universidade de São Paulo, Ribeirão Preto, SP, Brasil \\ ${ }^{4}$ Divisão de Ortopedia e Traumatologia, \\ Faculdade de Medicina de Ribeirão Preto, Universidade de São Paulo, \\ Ribeirão Preto, SP, Brasil \\ Corresponding author: M.S. Brassesco \\ E-mail: marsol@rge.fmrp.usp.br/solbrassesco@hotmail.com
}

Genet. Mol. Res. 8 (4): 1211-1217 (2009)

Received July 10, 2009

Accepted August 8, 2009

Published October 6, 2009

\begin{abstract}
Epithelioid sarcoma is a rare, aggressive soft tissue tumor of unknown histogenesis showing predominantly epithelioid cytomorphology. We conducted a conventional and molecular cytogenetic study of a 27-year-old male with epithelioid sarcoma with angiomatoid features. Cytogenetic analysis of epithelioid sarcoma metaphase spreads by GTG-banding revealed a diploid chromosome complement with structural and numerical aberrations. Comparative genomic hybridization analysis demonstrated the amplification of 3p24 pter, 4p15.2-p16 and 18q23, while chromosome losses involved 3p13-p14, 3q24-q26.1, 9q21, and 11q21. Fluorescence in situ hybridization assessment showed normal hybridization patterns for the $C$ $M Y C$ and CCND1 loci; CCND1 RNA overexpression was detected
\end{abstract}


by real-time polymerase chain reaction analysis. Genetic evaluation of this rare condition may be useful in determining if epithelioid sarcoma is associated with a distinct genetic background.

Key words: Cytogenetics; Epithelioid sarcoma; Cancer; Angiomatoid features

\section{INTRODUCTION}

Epithelioid sarcoma (ES) is an aggressive soft tissue tumor of unknown histogenesis showing predominantly epithelioid cytomorphology and affecting mainly young individuals between 10 and 39 years of age (Kaddu et al., 2008). Two main clinicopathologic types are recognized. The classic type manifests clinically as a painless, slow-growing nodule arising within the dermis or subcutis or, less commonly, in deep fascial or tenosynovial tissue. Usually, it occurs at the flexor surface of the fingers, hand, wrist, and forearm, followed by knee and lower leg and proximal extremities. On the other hand, the less common type, also known as the proximal variant or large cell/rhabdoid ES, occurs predominantly in adults in axial proximal locations such as pelvis, mediastinum and trunk, as deep infiltrative masses (Guillou and Kaneko, 2002). Furthermore, other less common variants have been described, including the angiomatoid variant, which features cyst formation and hemorrhage within tumor nodules (Laskin and Miettinen, 2003), and the fibroma-like variant (Fleury Jr. and Sanches Jr., 2006).

The genetic background of ES is poorly understood, and due to its rarity, cytogenetic data are scarce. While no consistent chromosomal aberrations have been specifically associated with ES, it has been suggested that deletions of $1 \mathrm{p}$ (Stenman et al., 1990) and alterations on chromosomes 8 and 22 could be involved in its tumorigenesis (Sonobe et al., 1997), as well as amplifications of C-MYC (Lualdi et al., 2004) and cyclin D1 gene (CCND1) (Lushnikova et al., 2000) located at 8q24 and 11q13, respectively. Furthermore, numerical aberrations together with $18 \mathrm{q}$ aberrations have also been described (Iwasaki et al., 1996). The present article describes the cytogenetic findings of ES with angiomatoid features (ESA) occurring in a young adult.

\section{MATERIAL AND METHODS}

A 27-year-old male was referred for investigation of a bulky, slow-growing round palpable mass in the palm of his right hand. The lesion was first detected seven months before admission as a small pale nodule that appeared after a traumatic injury to the area. The patient suffered from moderate pain and a region of ulceration in the skin above the mass became visible in the last two months; the patient also reported dyspnea and cough in the same period. One month before admission he was seen by a general physician who detected, besides the large skin lesion, a pneumothorax. He was subsequently referred to our institution for diagnostic investigation. There was no history of fever or weight loss in this period.

On physical examination, several firm and round palpable masses of various sizes (ranging from 1 to $6 \mathrm{~cm}$ ) were found in the right hand, arm and elbow. In addition, three large and highly vascularized ulcers, with internal necrotic aspect, were seen in the palm of his right hand (Figure 1). The complete blood cell count was normal and the erythrocyte sedimentation rate was slightly elevated. Chest X-ray and computed tomography scan showed several 
peripheral nodular images, leading to the hypothesis of pulmonary metastasis. A pulmonary transbronchial biopsy was performed and was negative for tumor cells.
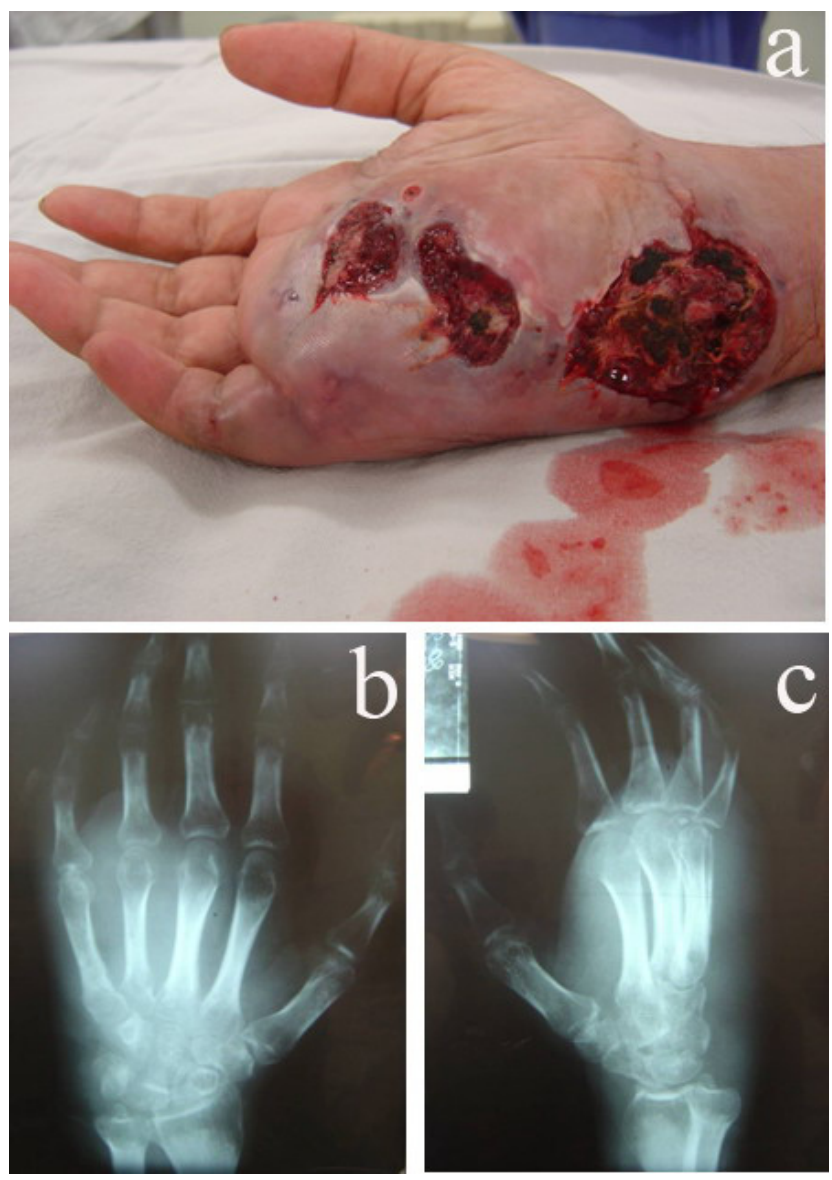

Figure 1. a. Gross aspect of the lesions. Note the intense swelling of the right hand, with strong vascularization, and multiple and deep-ulcerated skin lesions. b. and c. Lateral and anteroposterior radiograph showing the bulky extension of the soft tissues affected by the lesion.

The patient was submitted to surgical amputation of the right hand. The pathological exam of the specimen showed a highly malignant and infiltrative neoplasia, composed of fusiform and epithelioid cells, permeated by numerous immature blood vessels. Some areas of hemorrhage, necrosis and inflammation were also present. Mitotic figures were abundant. Immunohistochemical $(\mathrm{IH})$ staining was positive for CA125, epithelial membrane antigen (EMA), vimentin, and low- and high-molecular weight cytokeratins; focal positivity was observed for CD34, CD1A, and CD31 (all from Dako, Glostrup, Denmark) (Figure 2). IH staining was negative for desmin and collagen IV. The morphological and IH findings were consistent with ESA. 


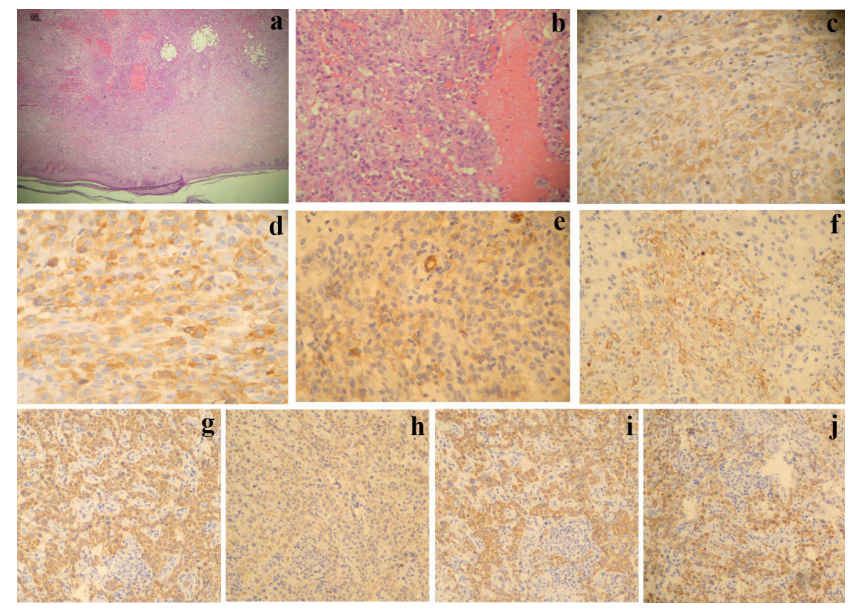

Figure 2. Histopathological aspect of the lesion. a. and b. Predominant pattern of the specimen (hematoxylin and eosin - 40X and 100X magnification, respectively). Note the hemorrhagic areas. c. Positivity for vimentin. $\mathbf{d}$. Positivity for epithelial membrane antigen. e. Focal CD34. f. Smooth muscle actin labeling. g-j. Positivity for lowand high-molecular weight cytokeratins: AE1/AE3, 34 $\beta, 35 \beta$, and CK7, respectively.

The patient was treated with chemotherapy $\left(1.5 \mathrm{~g} / \mathrm{m}^{2}\right.$ ifosfamide D1-D4; $30 \mathrm{mg} / \mathrm{m}^{2}$ doxorubicin D1-D3 and $5 \mu \mathrm{g} / \mathrm{kg}$ filgrastim D5-D14). He developed an acute respiratory failure and eventually died from infectious and pulmonary complications three weeks following the first cycle of chemotherapy.

Cytogenetic preparations from fresh tumor sample (adjacent to areas verified by frozen section) were obtained as previously described by Brassesco et al. (2009). The subsequent analysis was performed by GTG-banding, and the results were interpreted according to the International System for Human Cytogenetic Nomenclature 2005 guidelines (Shaffer and Tommerup, 2005).

Fluorescence in situ hybridization (FISH) analyses were also performed on the same cell preparations using commercially available probes for the CCND1 genes (LSI IGH/CCNDI XT dual-color, Abbott Vysis, Downers, IL, USA), and C-MYC genes (LSI IGH/CMYC dualcolor, Abbott Vysis) according to the protocol provided by the manufacturer. The preparations were dehydrated, dried, and counterstained with DAPI (Sigma Chemical Co., St. Louis, USA) plus antifade and analyzed with an epifluorescence microscope.

Comparative genome hybridization (CGH) was performed as described by Brassesco et al. (2009). Images were analyzed using an epifluorescence microscope (Olympus BX-40) equipped with a cooled CCD camera. Ratio profiles were calculated using the CGHView ${ }^{\text {TM }}$ EXPO software (Applied Spectral Imaging, Carlsbad, CA, USA).

For quantitative analysis of CCND1 (cyclin D1) gene expression, we used quantitative real-time polymerase chain reaction (PCR) - TaqMan probes (Assays-on-Demand; Applied Biosystems). Granta-519 cell line was used as the calibrator and the normalization factor was the geometric mean of the glyceraldehyde-3-phosphate dehydrogenase gene. The results for relative gene expression were analyzed using the $2^{-\Delta \Delta C \mathrm{CT}}$ method (Livak and Schmittgen, 2001). 


\section{RESULTS}

Twenty-nine metaphase cells were cytogenetically examined by GTG-banding, 11 of which showed a normal male chromosomal complement. The other 18 cells were karyotypically abnormal and characterized as shown in Table 1.

Table 1. Cytogenetic findings detected by GTG-banding in epithelioid sarcoma with angiomatoid features.

\begin{tabular}{lc}
\hline Cell & Karyotype \\
\hline $1-11$ & $46, \mathrm{XY}$ \\
12 & $44, \mathrm{XY},-8,-12$ \\
13 & $46, \mathrm{XY}, \mathrm{t}(8 ; 20)(\mathrm{q} 24 ; \mathrm{p} 11.1)$ \\
14 & $46, \mathrm{XY}, \mathrm{t}(3 ; 8)(\mathrm{p} 23 ; \mathrm{q} 24)$ \\
15 & $43, \mathrm{XY},-2 \mathrm{q},-7,-9,-9,-10,-15,-20,+22,+2 \mathrm{mar}$ \\
16 & $47, \mathrm{XY},-3 \mathrm{q},-16,+20+$ mar \\
17 & $44, \mathrm{XY},-6,-7,-8+\mathrm{mar}$ \\
18 & $46, \mathrm{XY},-21,+\mathrm{ace}$ \\
19 & $46, \mathrm{XY},-9,+\mathrm{mar}$ \\
20 & $46, \mathrm{XY}, \mathrm{del}(6)(\mathrm{q} 24-\mathrm{q} 27),+\mathrm{ace}$ \\
21 & $46, \mathrm{XY},-13+\mathrm{ace}$ \\
22 & $47, \mathrm{XY},-9,+2 \mathrm{mar}$ \\
23 & $46, \mathrm{XY}, \mathrm{t}(12 ; 17)(\mathrm{q} 24.3 ; \mathrm{q} 21)$ \\
24 & $46, \mathrm{XY}, \mathrm{t}(11,17)(\mathrm{q} 25 ; \mathrm{q} 21)$ \\
25 & $46, \mathrm{XY}, \mathrm{del}(7)(\mathrm{q} 36)$ \\
26 & $46, \mathrm{XY},-9,-9,+2 \mathrm{mar}$ \\
27 & $44, \mathrm{XY},-19,-20$ \\
28 & $45, \mathrm{XY}, \mathrm{add}(12)(\mathrm{q} 24.3)$ \\
29 & $45, \mathrm{XY},-2$ \\
\hline
\end{tabular}

Fifteen metaphases were analyzed by $\mathrm{CGH}$. This approach demonstrated the amplification of 3p24 pter, 4p15.2-p16, and 18q23, while chromosome losses involved 3p13-p14, 3q24-q26.1, 9q21, and 11q21 (Figure 3a). Dual-color FISH showed normal hybridization patterns for the $C-M Y C$ and $C C N D 1$ loci at $8 \mathrm{q} 24$ and 11q13, respectively (Figure $3 \mathrm{~b}$ and c). Relative gene expression analysis of the sample by quantitative real-time reverse transcriptase PCR, however, revealed an approximately 10-fold increase in the expression of the cyclin D1 gene (0.107) compared to normal skin (0.016) (data not shown).

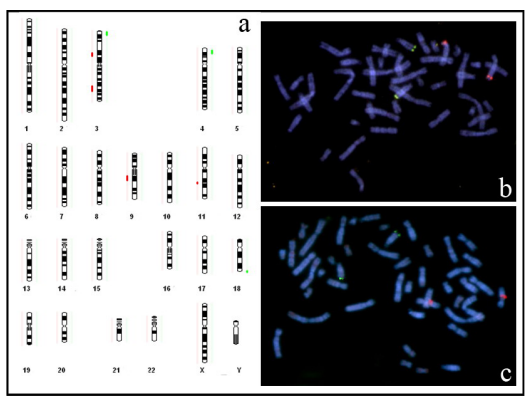

Figure 3. a. Schematic representation of gains and losses observed by comparative genome hybridization. Gains and losses are represented by lines on the right (green) and left (red) side of each chromosome ideogram, respectively. b. FISH with $I G H / C-M Y C$ dual-color probes showing the presence of two $I G H$ copies at $14 \mathrm{q} 32$ (SpectrumGreen), and two copies of $C-M Y C$ gene (SpectrumOrange) located at 8q24. c. FISH with IGH/CCND1 dual-color probes showing the presence of two IGH copies at 14q32 (SpectrumGreen), and two copies of cyclin DI gene (SpectrumOrange) located at 11q13. 


\section{DISCUSSION}

The genetic information on ES is limited to isolated case studies. Cytogenetically, no consistent chromosomal aberrations have been specifically attributed to this entity. Different studies on classic and proximal types have revealed structural and numerical aberrations involving chromosomes 8 and 22 (Cordoba et al., 1994; Sonobe et al., 1997; Lee et al., 2004); however, little is known about other less common types of ES. To our knowledge, the cytogenetic data on the angiomatoid variant in the literature is restricted to only three cases, all of which were retrospectively studied by CGH (Lushnikova et al., 2000).

In the present article, we report the cytogenetic findings of a 27 -year-old male with ESA. Cytogenetic analysis of metaphase spreads of the angiomatoid variant of ES at the time of diagnosis, using GTG-banding, revealed a diploid chromosome complement. However, $65 \%$ of cells showed non-clonal structural and numerical abnormalities. Two metaphases showed the involvement of $8 \mathrm{q} 24$, a chromosome band that contains the $C-M Y C$ gene, in structural aberrations. Gains of $8 \mathrm{q}$ and unbalanced cryptic translocations involving chromosome 8 with gains of $C-M Y C$ have been previously described in the proximal type ES (Lualdi et al., 2004), suggesting that overrepresentation of this gene may be a relevant event in epithelioid sarcomas. Nonetheless, no rearrangements involving this gene were detected by FISH with specific probes directed to $C-M Y C$ gene in the present case. The cytogenetic analysis also showed a lack of rearrangements involving chromosome 22. Interestingly, our analysis by GTG-banding showed loss of chromosome 9 in four cells, but the losses were accompanied by marker chromosomes, pointing to the possibility of this chromosome being rearranged, which cannot be detected by CGH.

The analysis of our tumor sample by CGH demonstrated gains of 3p24 pter, 4p15.2-p16, and 18q23, while chromosome losses involved 3p13-p14, 3q24-q26.1, 9q21, and 11q21. Lushnikova et al. (2000) reported DNA copy number changes in two of the three angiomatoid ES analyzed by the same technique, showing high amplification of $6 \mathrm{p} 21.3-$ p21.1 in one of the cases and gain in 11q12-21 in the other. These authors also found amplification of $11 \mathrm{q} 13$ to be the most common recurrent gain in both classic cases, angiomatoid and rhabdoid variants of ES, with increased cyclin D1 gene expression detected at the protein level (immunohistochemistry). The CGH and FISH assessment in our sample showed normal hybridization patterns and no chromosomal structural changes, with only two copies of this gene identified by these techniques. Interestingly, an overexpression of CCND1 ( 10fold increase) was detected by quantitative PCR, pointing to a higher expression of cyclin D1 gene at the RNA level. The molecular events underlying this genetic observation in the angiomatoid variant of ES deserve further investigation.

Interestingly, our CGH results showed loss of 3p13-14, which was also described by Lushnikova et al. (2000), suggesting that this region could harbor tumor suppressor genes relevant to the tumorigenesis of ES. Losses of heterozygosity on 3p13-3p21 are common in cervical carcinomas and can be observed in intra-epithelial lesions accompanying cervical cancers (Fouret et al., 1998) and vulvar carcinoma (Raitanen et al., 2004). Also, deletions of 3p13-p14 have been associated with immortalization in human uroepithelial cells (Vieten et al., 1998).

To date, the cytogenetic information on ES with angiomatoid features is scanty and limited to three cases in a single study. Additional reports may gather genetic information and background in order to determine if this variant of ES possesses distinct genetic characteristics. 


\section{ACKNOWLEDGMENTS}

The authors are grateful to Dr. Amanda Favero for photography assistance. We also thank the Pathology Department for diagnostic support. Research supported by Fundação de Amparo à Pesquisa do Estado de São Paulo (FAPESP \#06/04827-3 and \#07/54236-4).

\section{REFERENCES}

Brassesco MS, Valera ET, Neder L, Castro-Gamero AM, et al. (2009). Polyploidy in atypical grade II choroid plexus papilloma of the posterior fossa. Neuropathology 29: 293-298.

Cordoba JC, Parham DM, Meyer WH and Douglass EC (1994). A new cytogenetic finding in an epithelioid sarcoma, t(8;22)(q22;q11). Cancer Genet. Cytogenet. 72: 151-154.

Fleury LFF Jr and Sanches JA Jr (2006). Primary cutaneous sarcomas. An. Bras. Dermatol. 81: 207-221.

Fouret PJ, Dabit D, Mergui JL and Uzan S (1998). Loss of heterozygosity on the short arm of chromosome 3 in cervical intra-epithelial neoplasia without concomitant cervical carcinoma. Pathobiology 66: 306-310.

Guillou L and Kaneko Y (2002). Epithelioid Sarcoma. In: Pathology and Genetics of Tumors of Soft Tissue and Bone (Fletcher CDM, Unni KK and Mertens F, eds.). International Agency for Research on Câncer (IARC) Press, Lyon, 205-207.

Iwasaki H, Ohjimi Y, Ishiguro M, Isayama T, et al. (1996). Epithelioid sarcoma with an 18q aberration. Cancer Genet. Cytogenet. 91: 46-52.

Kaddu S, Wolf I, Horn M and Kerl H (2008). Epithelioid sarcoma with angiomatoid features: report of an unusual case arising in an elderly patient within a burn scar. J. Cutan. Pathol. 35: 324-328.

Laskin WB and Miettinen M (2003). Epithelioid sarcoma: new insights based on an extended immunohistochemical analysis. Arch. Pathol. Lab. Med. 127: 1161-1168.

Lee MW, Jee KJ, Ro JY, Lee DP, et al. (2004). Proximal-type epithelioid sarcoma: case report and result of comparative genomic hybridization. J. Cutan. Pathol. 31: 67-71.

Livak KJ and Schmittgen TD (2001). Analysis of relative gene expression data using real-time quantitative PCR and the 2(-Delta Delta C(T)) method. Methods 25: 402-408.

Lualdi E, Modena P, Debiec-Rychter M, Pedeutour F, et al. (2004). Molecular cytogenetic characterization of proximaltype epithelioid sarcoma. Genes Chromosomes Cancer 41: 283-290.

Lushnikova T, Knuutila S and Miettinen M (2000). DNA copy number changes in epithelioid sarcoma and its variants: a comparative genomic hybridization study. Mod. Pathol. 13: 1092-1096.

Raitanen M, Worsham MJ, Lakkala T, Carey TE, et al. (2004). Characterization of 10 vulvar carcinoma cell lines by karyotyping, comparative genomic hybridization and flow cytometry. Gynecol. Oncol. 93: 155-163.

Shaffer LG and Tommerup N (Editors) (2005). An International System for Human Cytogenetic Nomenclature (ISCN) 2005. S. Karger, Basel.

Sonobe H, Ohtsuki Y, Sugimoto T and Shimizu K (1997). Involvement of 8q, 22q, and monosomy 21 in an epithelioid sarcoma. Cancer Genet. Cytogenet. 96: 178-180.

Stenman G, Kindblom LG, Willems J and Angervall L (1990). A cell culture, chromosomal and quantitative DNA analysis of a metastatic epithelioid sarcoma. Deletion $1 \mathrm{p}$, a possible primary chromosomal abnormality in epithelioid sarcoma. Cancer 65: 2006-2013.

Vieten L, Belair CD, Savelieva L, Julicher K, et al. (1998). Minimal deletion of 3p13 $\rightarrow 14.2$ associated with immortalization of human uroepithelial cells. Genes Chromosomes Cancer 21: 39-48. 